\title{
Atividade antiinflamatória do extrato etanólico de Conocliniopsis prasiifolia R.M. King \& H. Robinson na resposta celular de neutrófilos
}

\author{
Maria das Graças da Silva,* Fernando de Sousa Oliveira, Margareth de Fátima Formiga \\ Melo Diniz, Orlando Seiko Takemura
}

Laboratório de Tecnologia Farmacêutica "Prof. Delby Fernandes de Medeiros", Universidade Federal da Paraíba, Caixa Postal 5009, 58051-970 João Pessoa-PB, Brasil

\begin{abstract}
RESUMO: Conocliniopsis prasiifolia (DC) R.M. King \& H. Robinson é uma espécie utilizada na medicina popular como antiinflamatória e analgésica, pertencente à família Asteraceae e conhecida como "mentrasto-roxo". Estudos prévios com o extrato etanólico das folhas de $C$. prasiifolia (EECp) demonstraram uma baixa toxicidade por via oral e intraperitoneal, bem como efeito analgésico e antiinflamatório em modelos animais. O presente estudo objetivou avaliar o efeito de EECp frente à degranulação de neutrófilos, e dessa forma melhor caracterizar o efeito antiinflamatório dessa espécie vegetal. Para tanto, foram utilizados neutrófilos peritoneais de ratos e técnicas espectrofotométricas. Os resultados demonstraram que EECp, nas concentrações de 25 e $50 \mu \mathrm{g} / \mathrm{mL}$, foi capaz de inibir de forma significativa $(\mathrm{p}<0,05)$ a degranulação de neutrófilos induzida por fMLP. Portanto, o EECp impede a liberação de grânulos dos neutrófilos contendo enzimas degradativas, demonstrando atividade antiinflamatória.
\end{abstract}

Unitermos: Conocliniopsis prasiifolia, Asteraceae, atividade antiinflamatória, degranulação de neutrófilos, flavonóide.

\begin{abstract}
Anti-inflammatory activity of the ethanol extract of Conocliniopsis prasiifolia R.M. King \& H. Robinson in the neutrophils cellular response”. Conocliniopsis prasiifolia (DC) R.M. King \& H. Robinson is a species used in popular medicine with anti-inflammatory and analgesic activities, it belongs to the Asteraceae family and is known as "mentrasto-roxo". Studies of the ethanol extract from its leaves (EECp) showed a low toxicity throughout oral and intraperitoneal routes, and anti-inflammatory and analgesic effects in animal models. The aim of this study was to evaluate the effect of EECp in the neutrophils degranulation for better characterization of its antiinflammatory effect. Peritoneal neutrophils from rats and spectrophotometric techniques were used. The results showed that the EECp $(25$ and $50 \mu \mathrm{g} / \mathrm{mL})$ was able to inhibit significantly $(\mathrm{p}<0.05)$ the neutrophils degranulation induced from fMLP. We can conclude that the EECp prevents the release of granules of the neutrophils that contains degrading enzymes, demonstrating anti-inflammatory activity.
\end{abstract}

Keywords: Conocliniopsis prasiifolia, Asteraceae, anti-inflammatory activity, neutrophils degranulation, flavonoid.

\section{INTRODUÇÃO}

A etnofarmacologia associada à descoberta de novas drogas, usando produtos naturais, é um assunto de grande importância no cenário mundial na atualidade (Patwardhan et al., 2004; Albuquerque \& Hanazaki, 2006). Entretanto, apesar do avanço tecnológico atual, o processo de descoberta de novas drogas que possam ser utilizadas na terapêutica é considerado o principal problema que afeta a indústria farmacêutica (Patwardhan, 2005).

Em vários registros famosos da antiguidade, tais como os de Hipócrates, Avicena e Paracelso, as plantas medicinais ocupavam lugar de destaque em práticas terapêuticas (Botsaris \& Machado, 1999). Ao longo dos séculos produtos de origem vegetal constituíram as bases de tratamento de diferentes doenças. A partir de plantas descritas e utilizadas na medicina popular foram descobertos diversos medicamentos usados até hoje na terapêutica tradicional (Almeida et al., 2001; Silva et al., 2003; Rocha et al., 2005; Amaral et al., 2006; BarbosaFilho et al., 2007; Oliveira et al., 2007; Saúde-Guimarães \& Faria, 2007; Barbosa-Filho et al., 2008; ).

Segundo a Organização Mundial de Saúde, cerca de $80 \%$ da população nos países em desenvolvimento faz uso de plantas medicinais no tratamento de doenças primárias (Carvalho et al., 2006; Carvalho et al., 2008). Neste sentido, inúmeros grupos de pesquisa em todo mundo têm voltado sua atenção para a validação do uso de plantas medicinais e o isolamento de seus metabólitos secundários, já que representam um importante recurso terapêutico desde os primórdios da humanidade até dias 
atuais.

Conocliniopsis prasiifolia (DC) R.M. King \& H. Robinson, é uma espécie pertencente à família Asteraceae, distribuída pela América do Sul, e utilizada na medicinal tradicional como antiinflamatória, analgésica e antipirética, sendo conhecida popularmente como "mentrasto-roxo", "aleluia" e "cabeça-de-lagartixa" (Matos, 1997; Agra et al., 2007 \& 2008). Caracteriza-se por ser uma planta com porte de subarbusto a arbusto, ereto e ramificado, com caule e ramos laxo-pilosos, folhas opostas de lâmina deltóide a lanceolada, margem irregularmente denteada a crenada. Inflorescências compostas em corimbos a panículas de capítulos, flores isomorfas, tubulosas, andróginas de coloração azul a lilás (Silva, 2001).

Estudo químico com as raízes de $C$. prasiifolia resultou no isolamento de noves lactonas sesquiterpênicas e uma cumarina (Bohlmann et al., 1980). Investigação química com as partes aéreas, levou a obtenção de lactonas sesquiterpênicas: conoprasiolido, seuacetato 5'- $O$-acetato, dois outros derivados, o tiglato e conoprasiolido-9- $O$, 5'-O-diacetato. Mais recentemente foram obtidas duas novas lactonas sesquiterpênicas bejaranolidos, $9 \beta$-hidroxi4E-bejaranolido e $3 \alpha$, $9 \beta$-dihidroxi-4E-bejaranolido; dois furoheliangolidos, conoprasiolido-9- $O, 5$ '- $O$-diacetato e 5'-desoxiconoprasiolido e um cromeno (Bohlmann et al., 1984). Macha sistemática para isolamento dos constituintes das partes aéreas de C. prasiifolia, resultou na obtenção de quatro flavonóides: o 5-hidróxi4",7-dimetoxiflavona; 4',5,6,7-tetrametoxiflavona; 4',5,7-trimetoxiflvona e 4',5-diidróxi-7-metoxiflavona, todos esses descritos como inéditos para a espécie (Almeida et al., 2006). Estudos prévios recentes com o extrato etanólico das folhas $C$. prasiifolia demonstrou a baixa toxicidade por via oral e intraperitoneal em camundongos, além do efeito antinociceptivo e antiinflamatório dessa espécie em modelos animais (Silva et al., 2005).

Com base nesses dados e para complementar o estudo farmacológico de $C$. prasiifolia, o presente trabalho objetivou investigar o efeito de $C$. prasiifolia na degranulação de neutrófilos peritoneais de ratos, e dessa forma, melhor caracterizar a atividade antiinflamatória de C. prasiifolia em roedores.

\section{MATERIAL E MÉTODOS}

\section{Material botânico}

As folhas de C. prasiifolia (DC) R.M. King \& H. Robinson foram coletadas na região do município de Santa Rita / Paraíba / Brasil em outubro de 2001. O material botânico dessa espécie foi identificado pela $\mathrm{Dr}^{\mathrm{a}}$ Maria de Fátima Agra da Universidade Federal da Paraíba, sendo uma exsicata depositada no Herbário Lauro Pires Xavier do Laboratório de Tecnologia Farmacêutica sob o código - (M F Agra 2052 JPB, LTF).

\section{Obtenção do extrato}

As folhas previamente selecionadas foram secas em estufa a $40^{\circ} \mathrm{C}$ e submetidas à maceração com etanol a $95 \%$, por 72 horas. Após este período foi feita a filtração e a concentração do extrato em evaporador rotatório, sendo em seguida liofilizado obtendo-se o extrato etanólico das folhas de Conocliniopsis prasiifolia (EECp). Para realização do ensaio biológico, o EECp foi dissolvido em dimetilsulfóxido (DMSO), concentração não superior a $0,05 \%$.

\section{Animais}

Foram utilizados ratos albinos machos da espécie Ratus norvegicus, linhagem Wistar, pesando 200 - 300 g, com aproximadamente três meses de vida, sendo fornecidos pelo Biotério Prof. Dr. Thomas George da Universidade Federal da Paraíba. Os animais foram mantidos sob condições controladas de temperatura (21 $\pm 1{ }^{\circ} \mathrm{C}$ ) e ciclo de claro/escuro de 12 horas, tendo a fase clara iniciando-se às $6 \mathrm{~h}$ e terminando às $18 \mathrm{~h}$, com livre acesso a água e submetidos a um jejum de 12 horas antes dos experimentos.

\section{Obtenção de neutrófilos peritoneais de rato}

Para a obtenção de neutrófilos da cavidade peritoneal de ratos, foi administrado $10 \mathrm{~mL}$ de solução glicogenada a $0,2 \%$ em salina na cavidade peritoneal de ratos Wistar. Decorrido 4 horas, foi realizada a coleta das células por lavagem da cavidade peritoneal com solução tampão de neutrófilos (HBSS-HEPES) contendo heparina (1000 U). Em seguida, o material coletado foi centrifugado $\left(250 \mathrm{~g} / 6 \mathrm{~min}\right.$ a $\left.5{ }^{\circ} \mathrm{C}\right)$. Possíveis eritrócitos contaminantes foram eliminados através de choque hipotônico utilizando-se $5 \mathrm{~mL}$ de solução de $\mathrm{NaCl}$ a $0,2 \%$ e após 20 segundos, adicionou-se $5 \mathrm{~mL}$ de solução de $\mathrm{NaCl}$ a 1,6\%. Logo após, as células foram lavadas e mantidas até o uso, em soluções tampões para neutrófilos (Kanaho et al., 1993; Nakashima et al., 1995; Takemura et al., 1997).

A checagem da pureza, viabilidade e o ajustamento do número de células por ensaio, foram feitos por contagem em retículo de Neubauer ao microscópio utilizando solução corante de Trypan blue a $0,4 \%$.

\section{Ensaios de degranulação em neutrófilos}

Os neutrófilos obtidos pela lavagem peritoneal foram utilizados nos experimentos de degranulação. As células $\left(1 \times 10^{6}\right.$ células / tubo $)$ foram pré-incubadas com diferentes concentrações do EECp por 5 minutos.

Em seguida foram tratadas com citocalasina $\mathrm{B}$ $(5 \mu \mathrm{g} / \mathrm{mL})$ e estimuladas com peptídeo fMLP (N-formilmetionil-leucil-fenilalanina). Após 5 minutos, a reação foi finalizada com a adição de Buffer PBS frio ao tubo 
experimental e posterior centrifugação $(250 \mathrm{~g} / 6 \mathrm{~min}$ a $5{ }^{\circ} \mathrm{C}$ ). O sobrenadante (SOB-1) de cada tubo foi coletado em microtubos e as células resuspendidas com uma solução de PBS e detergente Triton x - 100 (0,01\%). Após rápida microcentrifugação (15000 RPM / $10 \mathrm{seg})$ o sobrenadante (SOB-2) foi coletado e acondicionado em freezer a $-25{ }^{\circ} \mathrm{C}$ até a determinação colorimétrica.

Foi avaliada a atividade celular a partir da quantidade de enzimas liberadas em condições de presença e ausência de estímulo. A atividade inibitória do extrato vegetal foi verificada comparando-se células tratadas e não tratadas (controle).

A quantificação por métodos espectrofotométricos $(415 \mathrm{~nm})$ foi feita com a reação de alíquotas do SOB-1 e SOB-2 com o substrato enzimático específico ( $p$-nitrofenil- $N$-acetil $\beta$-D-glucosaminida), inicialmente em solução tampão citrato $(\mathrm{pH} 4,5)$ e em seguida com solução tampão glicina ( $\mathrm{pH}$ 10,5) (Kanaho et al., 1993; Nakashima et al., 1995; Takemura et al., 1997). Como substância padrão foi utilizada a quercetina na concentração de $50 \mu \mathrm{M}$.

\section{Análise estatística}

Os resultados foram expressos em média \pm erro padrão da média (EPM). A análise estatística dos resultados foi realizada utilizando o teste " $t$ " de Student, sendo os resultados considerados significativos quando $\mathrm{p}$ $<0,05$.

\section{RESULTADOS}

De acordo com a Figura 1, nos ensaios biológicos in vitro realizados para determinação da atividade de EECp na resposta celular em neutrófilos, foi observada atividade antiinflamatória nas concentrações de 25 e 50 $\mu \mathrm{g} / \mathrm{mL}$, por inibir de forma significativa a degranulação em neutrófilos induzida por fMLP, demonstrando resultados semelhantes à quercetina $50 \mu \mathrm{M}$ utilizada como controle positivo.

\section{DISCUSSÃO E CONCLUSÃO}

Testes de bioatividade in vitro são ensaios que, na maioria das vezes, proporcionam rapidez e simplicidade na execução, aliados a outras vantagens como requerimento de quantidades mínimas das substâncias que se quer determinar a atividade. Estes experimentos tendem a fornecer informações valiosas sobre o efeito dos extratos vegetais nas células, como no caso das que estão envolvidas em processos inflamatórios agudos.

Foram realizados experimentos in vitro para determinação da atividade do EECp na resposta celular. Uma resposta efetiva do hospedeiro ao agente agressor requer um acúmulo local de leucócitos (Pettit \& Fredric, 1998). O tipo de leucócito que migra varia com a fase da lesão inflamatória e com o tipo de estímulo. Na maioria

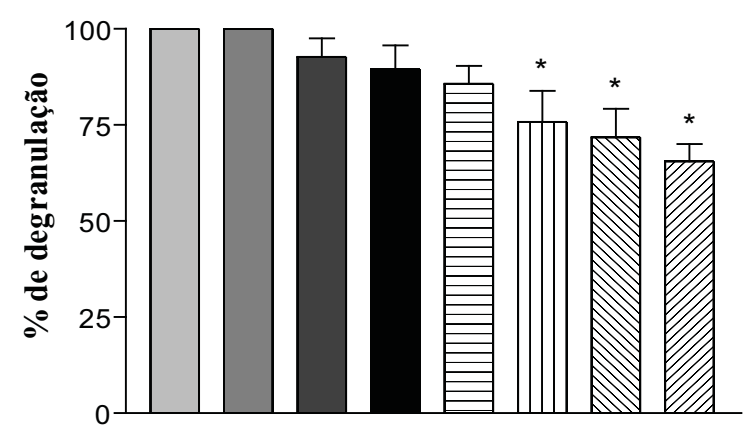

Tratamento

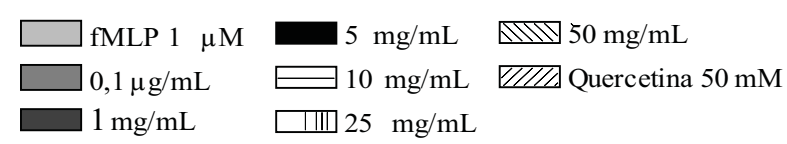

Figura 1. Percentagem de inibição de diferentes concentrações do extrato etanólico das folhas de Conocliniopsis prasiifolia e quercetina na degranulação de neutrófilos peritoneais de ratos estimulados com $N$-formil-metionil-leucil-fenilalanina. Os resultados expressam média \pm erro padrão da média, sendo $\mathrm{p}<0,05$ comparado com o grupo controle tratado com fMLP (Teste " $t$ " de Student / N=4 experimento-teste).

das vezes neutrófilos predominam nas primeiras 6 a 24 horas da fase aguda da reação inflamatória. Estas células fagocitam agentes lesivos, matam bactérias, degradam tecidos necróticos e antígenos estranhos (Bevilacqua et al., 1994).

Os ensaios biológicos realizados permitiram observar a resposta dos neutrófilos estimulados a liberar grânulos contendo enzimas degradativas de acordo com a técnica de Takemura et al. (1997).

Em todos os ensaios foi utilizado como controle positivo a quercetina $(50 \mu \mathrm{M})$, um flavonóide que inibe a degranulação de neutrófilos em torno de 30 a $50 \%$ (Takemura et al., 1997). O agonista utilizado fMLP, simula infecção bacteriana e estimula a resposta celular em neutrófilos. Em concentrações de EECp acima de 50 $\mu \mathrm{g} / \mathrm{mL}$, a própria cor do extrato interfere nos valores de absorbância e por isso não foram testados.

Foi constatada atividade antiinflamatória significativa do EECp nas concentrações de 25 e $50 \mu \mathrm{g} /$ $\mathrm{mL}$, já que este foi capaz de inibir a liberação enzimática de $\beta$-glucosaminidase, que foi a enzima lisossômica marcadora do processo de degranulação dos neutrófilos, utilizada nos ensaios.

É provável que a atividade antiinflamatória atribuída pela população a $C$. prasiifolia ora analisada, esteja relacionada com a presença de flavonóides, como por exemplo os descritos por Almeida et al. (2006). Esses compostos caracterizam-se do ponto de vista químico por apresentarem o núcleo fenilbenzopirano. Aos flavonóides são atribuídas propriedades farmacológicas, particularmente atividades antiinflamatória e analgésica (Emin et al., 1994). Essa atividade antiinflamatória está 
relacionada à capacidade desses de inibir o metabolismo do ácido araquidônico (Ferrandiz \& Alcaraz, 1991).

Portanto, a atividade antiinflamatória demonstrada nos ensaios biológicos in vitro foi corrobora com os resultados dos testes in vivo como demonstrados por Silva et al. (2005), uma vez que o extrato de $C$. prasiifolia inibiu de forma significativa a degranulação dos neutrófilos.

\section{AGRADECIMENTOS}

Os autores agradecem a José Crispim Duarte e Raimundo Nonato Filho pela assistência técnica, a $\mathrm{Dr}^{\mathrm{a}}$ Maria de Fátima Agra pela identificação botânica da espécie estudada e ao Conselho de Desenvolvimento Científico e Tecnológico (CNPq) pelo suporte financeiro.

\section{REFERENCIAS}

Agra MF, França PF, Barbosa-Filho JM 2007. Synopsis of the plants known as medicinal and poisonous in Northeast of Brazil. Rev Bras Farmacogn 17: 114-140.

Agra MF, Silva KN, Basílio IJLD, França PF, Barbosa-Filho JM 2008. Survey of medicinal plants used in the region Northeast of Brazil. Rev Bras Farmacogn 18: 472508.

Albuquerque UP, Hanazaki N 2006. As pesquisas etnodirigidas na descoberta de novos fármacos de interesse médico e farmacêutico: fragilidades e pespectivas. Rev Bras Farmacogn 16 (Supl.): 678-689.

Almeida RN, Navarro DS, Barbosa-Filho JM 2001. Plants with central analgesic activity. Phytomedicine 8: 310-322.

Almeida JRGS, Silva MS, Cunha EVL, Athaíde-Filho PF, Diniz MFFM, Silva MG, Takemura OS, Barbosa-Filho JM 2006. Chemical constituents and analgesic activity of Conocliniopsis prasiifolia. Pharm Biol 44: 76-78.

Amaral FMM, Ribeiro MNS, Barbosa-Filho JM, Reis AS, Nascimento FRF, Macedo RO 2006. Plants and chemical constituents with giardicidal activity. Rev Bras Farmacogn 16 (Supl.): 696-720.

Barbosa-Filho JM, Nascimento-Júnior FA, Tomaz ACA, Athayde-Filho PF, Silva MS, Cunha EVL, Souza MFV, Batista LM, Diniz MFFM 2007. Natural products with antileprotic activity. Rev Bras Farmacogn 17: 141148.

Barbosa-Filho JM, Alencar AA, Nunes XP, Tomaz ACA, Sena-Filho JG, Athayde-Filho PF, Silva MS, Souza MFV, da-Cunha EVL 2008. Sources of alpha-, beta, gamma-, delta- and epsilon-carotenes: A twentieth century review. Rev Bras Farmacogn 18: 135-154.

Bevilacqua MP, Nelson RM, Mannuri G 1994. Endhotelialleukocyte adhesion molecules in human disease. Ann Rev Med 45: 361-378.

Bohlmann F, Zdero C, King RM, Robinson H 1980. New heliangolides from Conocliniopsis prasiifolia. Phytochemistry 19: 1547-1549.

Bohlmann F, Zdero C, King RM, Robinson H 1984. Heliangolides and bejaranolides from Conocliniopsis prasiifolia. Phytochemistry 23: 1509-1511.

Botsaris AS, Machado PV 1999. Memento terapêutico fitoterápico. Rio de Janeiro: Flora Medicinal.

Carvalho ACB, Figueiredo CAV, Oliveira FS, Barbosa-Filho
JM, Oliveira, RAG 2006. As plantas medicinais e suas interações com medicamentos. In: Diniz, M.F.F.M, Oliveira, R.A.G., Medeiros, A.C.D., Malta Júnior, A., Moura, M.D. (org.) Memento de plantas medicinais. 1.ed. João Pessoa: Editora Universitária / UFPB, p.121-125.

Carvalho ACB, Balbino EE, Maciel A, Perfeito JPS 2008. Situação do registro de medicamentos fitoterápicos no Brasil. Rev Bras Farmacogn 18: 314-319.

Emin JAS, Oliveira AB, Lapa AJ 1994. Anti-inflammatory activity of hesperidin. $J$ Pharm Pharmacol 46: 118122.

Ferrandiz ML, Alcaraz MJ 1991. Anti-inflammatory activity and inhibition of arachidonic acid metabolism by flavonoids. Agents Actions 32: 283-288.

Kanaho Y, Nakai Y, Katoh M, Nozawa Y 1993. The phosphatase inhibitor 2,3 diphosphoglycerate interferes with phospholipase D activation in rabbit peritoneal neutrophils. J Biol Chem 268: 12492-12497.

Matos FJA 1997. Parte II: Conjunto de vertebes descritos das plantas medicinais do formulário therapêutico do professor Dias da Rocha. In: Matos, F.J.A. (org.) $O$ formulário fitoterápico do professor Dias da Rocha. 1.ed. Fortaleza: EUFC, p.49 - 226.

Nakashima S, Mizutani T, Nakanura Y, Takemura OS, Nakamura M, Miyata H, Katagiri Y, Nozawa Y 1995. Effects of selective phosphodiesterase type IV inhibitor, rolipran, on transducing phospholipases in neutrophils. Comp Biochem Physiol 112: 137-143.

Oliveira FQ, Gobira B, Guimarães C, Batista J, Barreto M, Souza M 2007. Espécies vegetais indicadas na odontologia. Rev Bras Farmacogn 17: 466-476.

Patwardhan B 2005. Ethnopharmacology and drug discovery. $J$ Ethnopharmacol 100: 50-52.

Patwardhan B, Vaidya ABD, Chorghade M 2004. Ayurveda and natural products drug discovery. Current Sci 86: 789799.

Pettit EJ, Fredric SF 1998. Cytosolic free calcium and the cytoskeleton in the control of leucocyte chemotaxis. Physiol Rev 78: 949-967.

Rocha LG, Almeida JRGS, Macedo RO, Barbosa-Filho JM 2005. A review of natural products with antileishmanial activity. Phytomedicine 12: 514-535.

Saúde-Guimarães DA, Faria AR 2007. Substâncias da natureza com atividade anti-Trypanosoma cruzi. Rev Bras Farmacogn 17: 455-465.

Silva MG 2001. Estudo das atividades antiinflamatória analgésica de Conocliniopsis prasiifolia (DC) R. M. King \& H. Robinson (Asteraceae). João Pessoa, 68p. Dissertação de Mestrado - Programa de Pós-graduação em Produtos Naturais e Sintéticos Bioativos, Universidade Federal da Paraíba.

Silva JS, Moura MD, Oliveira RAG, Diniz MFFM, BarbosaFilho JM 2003. Natural products inhibitors of ovarian neoplasia. Phytomedicine 10: 221-232.

Silva MG, Oliveira FS, Quintans-Júnior LJ, Oliveira TML, Diniz MFFM 2005. Investigação do efeito analgésico central e antiinflamatório de Conocliniopsis prasiifolia (DC) R.M. King \& H. Robinson em Roedores. Acta Farm Bonaerense 24: 533-537.

Takemura OS, Banno Y, Nozawa Y 1997. Inibition of $N$-formylmethionyl - leucyl - phenylalanine stimulated tyrosine phosphorylation and phospholipase D activation by quercetin in rabbit neutrophilis. Biochem Pharmacol 46: 103-107. 